Marquette University

e-Publications@Marquette

Biological Sciences Faculty Research and

Publications

Biological Sciences, Department of

6-1997

\title{
Effect of Intermittent Weight Bearing on Soleus Fiber Force- velocity-power and Force-pCa Relationships
}

J. J. Bangart

Marquette University

Jeffrey J. Widrick

Marquette University

Robert H. Fitts

Marquette University, robert.fitts@marquette.edu

Follow this and additional works at: https://epublications.marquette.edu/bio_fac

Part of the Biology Commons

\section{Recommended Citation}

Bangart, J. J.; Widrick, Jeffrey J.; and Fitts, Robert H., "Effect of Intermittent Weight Bearing on Soleus Fiber Force-velocity-power and Force-pCa Relationships" (1997). Biological Sciences Faculty Research and Publications. 451.

https://epublications.marquette.edu/bio_fac/451 
Marquette University

e-Publications@Marquette

\title{
Department of Biology Faculty Research and Publications/College of Arts and Sciences
}

This paper is NOT THE PUBLISHED VERSION; but the author's final, peer-reviewed manuscript. The published version may be accessed by following the link in the citation below.

Jounral of Applied Physiology, Vol. 82, No. 6 (1997, June): 1905-1910. DOI. This article is C American Physiological Society and permission has been granted for this version to appear in e-

Publications@Marquette. American Physiological Society does not grant permission for this article to be further copied/distributed or hosted elsewhere without the express permission from American

Physiological Society.

\section{Effect of Intermittent Weight Bearing on Soleus Fiber Force-velocity-power and Force- pCa Relationships}

\author{
J. J. Bangart \\ Department of Biology, Marquette University, Milwaukee, Wisconsin \\ J. J. Widrick \\ Department of Biology, Marquette University, Milwaukee, Wisconsin \\ R. H. Fitts \\ Department of Biology, Marquette University, Milwaukee, Wisconsin
}

\section{Abstract}

Bangart, J. J., J. J. Widrick, and R. H. Fitts. Effect of intermittent weight bearing on soleus fiber force-velocitypower and force-pCa relationships. J. Appl. Physiol. 82(6): 1905-1910, 1997.-Rat permeabilized type I soleus fibers displayed a $33 \%$ reduction in peak power output and a $36 \%$ increase in the free $\mathrm{Ca}^{2+}$ concentration required for one-half maximal activation after 14 days of hindlimb non-weight bearing (NWB). We examined the effectiveness of intermittent weight bearing (IWB; consisting of four 10-min periods of weight bearing/day) as a countermeasure to these functional changes. At peak power output, type I fibers from NWB animals produced 
$54 \%$ less force and shortened at a $56 \%$ greater velocity than did type I fibers from control weight-bearing animals while type I fibers from the IWB rats produced $26 \%$ more absolute force than did fibers from the NWB group and shortened at a velocity that was only $80 \%$ of the NWB group mean. As a result, no difference was observed in the average peak power of fibers from the IWB and NWB animals. Hill plot analysis of force-pCa relationships indicated that fibers from the IWB group required similar levels of free $\mathrm{Ca}^{2+}$ to reach half-maximal activation in comparison to fibers from the weight-bearing group. However, at forces $<50 \%$ of peak force, the force-pCa curve for fibers from the IWB animals clearly fell between the relationships observed for the other two groups. In summary, IWB treatments1) attenuated the NWB-induced reduction in fiber $\mathrm{Ca}^{2+}$ sensitivity but 2) failed to prevent the decline in peak power that occurs during NWB because of opposing effects on fiber force (an increase vs. NWB) and shortening velocity (a decrease vs. NWB).

THE REMOVAL of normal weight-bearing activity leads to rapid changes in the function of single fibers obtained from rat hindlimb extensor muscles. Soleus fibers expressing the type I myosin heavy chain (MHC) isoform are particularly susceptible to prolonged non-weight bearing. When activated with saturating levels of free $\mathrm{Ca}^{2+}$, chemically permeabilized type I soleus fibers from hindlimb-suspended rats display a substantial increase in their maximal unloaded shortening velocity $(\underline{8}, \underline{17}, \underline{23}, \underline{27})$. At relative loads less than $\sim 50 \%$ of peak isometric force, these fibers shorten at considerably greater velocities than do fibers obtained from weight-bearing animals (16). However, type I soleus fibers from non-weight-bearing animals produce considerably less absolute force than do fibers from weight-bearing controls $(\underline{8}, \underline{16}, \underline{17}, \underline{23}, \underline{27})$, and, as a result, their peak power is reduced by up to $50 \%(\underline{16})$.

Attention is now being directed to the development of procedures to attenuate or prevent non-weight-bearinginduced alterations in mammalian lower limb extensor muscle function. It is thought that these countermeasures will have important applications in the manned space program, where a reduction in voluntary ankle extensor muscle torque during isokinetic movements has been documented after spaceflight (13), and in ground-based clinical settings, where similar functional changes have been observed in human knee extensor performance after prolonged bed rest ()ㅡ.

One of the simplest countermeasures used to date has been to provide non-weight-bearing animals with brief, intermittent periods of standing weight support $(\underline{5}, \underline{22}, \underline{26}, \underline{27})$. Our laboratory (27) previously reported that four 10-min intermittent weight-bearing sessions per day during 14 days of hindlimb suspension attenuated changes in the peak isometric force and maximal unloaded shortening velocity (determined from slack test measurements) of single type I soleus fibers. However, isometric and/or unloaded contractions are not the predominant forms of muscle action during animal locomotion. Instead, muscle fibers shorten while under tension to produce the mechanical work and power required for movement. In the present study, we examined the effect of intermittent weight-bearing treatments on the isotonic contractile properties of type I soleus fibers.

The dependence of force development on $\mathrm{Ca}^{2+}$ is altered by prolonged non-weight bearing. Type I fibers from non-weight-bearing animals require a significantly greater free $\mathrm{Ca}^{2+}$ concentration than do fibers obtained from weight-bearing controls to attain $50 \%$ of their peak isometric force $(\underline{8}, \underline{18})$. Therefore, an additional objective of this study was to determine whether intermittent weight bearing attenuated this reduction in fiber $\mathrm{Ca}^{2+}$ sensitivity.

\section{METHODS}

This study was approved by the Animal Care Committee at Marquette University. Adult male Sprague-Dawley rats were divided into three groups: normal caged weight bearing, 14 days of non-weight bearing, and 14 days of intermittent weight bearing. Hindlimb weight-bearing activity of both the non-weight-bearing and the 
intermittent weight-bearing animals was eliminated by a tail-suspension procedure (27). Four times each day (at $0,4,8$, and $11.75 \mathrm{~h}$ of the light cycle), the intermittent weight-bearing animals were removed from hindlimb suspension and placed in an upright standing position on a horizontal surface. To prevent extensive ambulatory activity, a small wire enclosure was placed over each animal during the intermittent weight-bearing period. This enclosure was large enough to allow the animal to turn about but too small to allow walking activity. After 10 min of weight bearing, the intermittent weight-bearing animals were resuspended.

At the conclusion of the experimental treatments, soleus muscle bundles were prepared as previously described (27). These bundles were stored for up to 28 days at $-20^{\circ} \mathrm{C}$ in skinning solution containing (in $\mathrm{mM}$ ) $125 \mathrm{~K}$ propionate, 20 imidazole ( $\mathrm{pH} 7.0), 2$ ethylene glycol-bis( $\beta$-aminoethyl ether)- $N, N, N^{\prime}, N^{\prime}$-tetraacetic acid (EGTA), 4 ATP, and $1 \mathrm{MgCl}_{2}$ and $50 \%$ glycerol vol/vol.

The composition of the relaxing and $\mathrm{Ca}^{2+}$-activating solutions used for the single fiber functional experiments was determined by using the computer program of Fabiato and Fabiato (ㄱ) and the apparent stability constants (corrected for temperature, $\mathrm{pH}$, and ionic strength) reported by Godt and Lindley (10). Each solution contained (in mM): 7 EGTA, 20 imidazole, 10 caffeine, 14.5 creatine phosphate, 1 free $\mathrm{Mg}^{2+}$, and 4 free MgATP. The relaxing solution contained a free $\mathrm{Ca}^{2+}$ concentration of pCa 9.0 (where $\mathrm{pCa}$ is $-\log \mathrm{Ca}^{2+}$ concentration) and the activating solution a free $\mathrm{Ca}^{2+}$ concentration of $\mathrm{pCa} 4.5$. Sufficient $\mathrm{KOH}$ and $\mathrm{KCl}$ were present in both solutions to achieve a pH of 7.0 and a total ionic strength of $180 \mathrm{mmol} / \mathrm{l}$. Submaximal activating solutions, ranging from $\mathrm{pCa}$ 7.0 to 4.7 , were made by mixing appropriate volumes of relaxing and maximal activating solutions to attain the required free $\mathrm{Ca}^{2+}$ concentration.

On the day of an experiment, a muscle bundle was transferred into cold relaxing solution, and a single soleus fiber segment was isolated, transferred into an experimental chamber filled with relaxing solution, and mounted between an isometric force transducer (model 400, Cambridge Technology, Watertown MA) and a directcurrent position motor (model 300B, Cambridge Technology) by using small monofilament posts and 10.0 suture. Sarcomere length was adjusted to $2.5 \mu \mathrm{m}$ by using an eyepiece micrometer $(\times 800)$. The length of the fiber was measured and recorded. A photograph was taken of the fiber while it was briefly suspended in air. Fiber width was determined at three points along the length of the photo. The mean of these measurements was taken as fiber diameter and used to calculate fiber cross-sectional area under the assumption that the fiber forms a circular cross section when suspended in air (19). The fiber was briefly bathed in relaxing solution containing 0.5\% (wt/vol) Brij 58 (polyoxyethylene 20 cetyl ether, Sigma Chemical, St. Louis, MO) to inhibit any sarcoplasmic reticulum $\mathrm{Ca}^{2+}$ uptake activity. Detailed descriptions of the mounting procedure and experimental apparatus have been presented in previous papers $(\underline{8}, \underline{16}, \underline{27})$.

Contractions were induced by rapidly transferring the mounted fiber into an adjacent chamber filled with a $\mathrm{Ca}^{2+}-$ activating solution. Outputs from the force transducer and position motor were directed to a digital storage oscilloscope, amplified, and then interfaced to a personal computer via a Lab Master input-output board (Scientific Solutions, Solon, $\mathrm{OH}$ ). Activating and relaxing solutions were maintained at $15^{\circ} \mathrm{C}$ during all experiments.

All fibers were subjected to a series of isotonic contractions as follows. The fiber was activated in $\mathrm{pCa} 4.5$, and after peak isometric force was attained, the position motor, operating via a servomechanism similar to that described by Julian and Moss (15), stepped the fiber to three submaximal isotonic loads. Each isotonic contraction lasted between 100 and 150 ms. At the conclusion of the final isotonic load, the fiber was slackened to a length $\leq 20 \%$ of fiber length and was transferred back into relaxing solution where it was reextended to its original fiber length. The entire procedure was repeated so that each fiber was subjected to 15-18 different isotonic loads. Custom software determined the peak isometric force and the average force and average velocity over the final half of each isotonic step. The force baseline was defined as the resting force recorded while the 
fiber was in relaxing solution immediately before activation. Force-velocity data were fit with the hyperbolic Hill equation (14) by an iterative curve- fitting algorithm (Marquardt algorithm). For each individual fiber, the forcevelocity parameters $V_{\max }[y$-intercept of the relationship in fiber lengths/s $(\mathrm{FL} / \mathrm{s})], a / \mathrm{P}_{\mathrm{o}}$ (where $a$ is a constant with dimensions of force and $\mathrm{P}_{\mathrm{o}}$ is peak isometric force), and peak isometric force were used to calculate peak absolute power $\left(\mu \mathrm{N} \cdot \mathrm{FL} \cdot \mathrm{s}^{-1}\right)$ and peak normalized power $\left(\mathrm{kN} \cdot \mathrm{m}^{-2} \cdot \mathrm{FL} \cdot \mathrm{s}^{-1}\right)$. Force-velocity parameters were used to construct force-velocity and force-power relationships for each fiber (28). Velocity (and power) values corresponding to 101 separate force bins (0-100\% of peak isometric force) for each individual relationship were summed across fibers to obtain a single composite relationship for all fibers making up a particular group (28).

Force-pCa relationships were determined by recording peak isometric force during maximal (pCa 4.5) and submaximal ( $\mathrm{pCa}$ 6.8-4.7) $\mathrm{Ca}^{2+}$ activation. Periodic activations were conducted at $\mathrm{pCa} 4.5$ to monitor preparation viability. All submaximal forces $\left(P_{r}\right)$ were expressed relative to the peak force obtained at $p C a 4.5$; i.e., $P_{r}$ is ratio of peak force in given $\mathrm{pCa}$ to peak force in pCa 4.5. Force-pCa data were fit to Hill plots $\left\{\right.$ where $\log \left[P_{r} /\left(1-P_{r}\right)\right]$ is plotted against $\mathrm{pC}$ a by using least squares regression analysis. Two Hill plots were obtained for each fiber: one from data points $<0.5 \operatorname{Pr}$ (Hill plot coefficient $n_{2}$ ) and another using data points $>0.5 \operatorname{Pr}$ (Hill plot coefficient $n_{1}$ ). The value for the free $\mathrm{Ca}^{2+}$ concentration eliciting one-half maximal activation was calculated as the mean abscissal intercept of the plots. The $\mathrm{Ca}^{2+}$-activation threshold was calculated as the free $\mathrm{Ca}^{2+}$ concentration corresponding to an ordinal value of -2.5 for the Hill plot by using data points $<0.5 \mathrm{P}_{\mathrm{r}}$.

After the contractile measurements, the fiber was solubilized in $10 \mu \mathrm{l}$ of $1 \%$ sodium dodecyl sulfate sample buffer (for composition, see Ref. 27) and stored at $-80^{\circ} \mathrm{C}$. Later, $\sim 0.5 \mathrm{nl}$ of fiber volume was run on a Hoefer SE 600 gel-electrophoresis system, consisting of a $3 \%$ (wt/vol) acrylamide stacking gel and a 5\% (wt/vol) separating gel as previously described (27). Gels were silver stained according to the procedures described by Giulian et al. (9). Representative gels illustrating MHC identification in single rat soleus fibers have been presented in previous work from this laboratory $(\underline{8}, \underline{16}, \underline{27})$.

Results are presented as means \pm SE. A one-way analysis of variance was used to determine significant differences between the three experimental groups. When a significant $F$-ratio was obtained, the StudentNewman-Keuls post hoc procedure was used to determine differences between specific means. Statistical significance was accepted at $P<0.05$.

\section{RESULTS}

\section{Body and muscle mass.}

There was no difference in the average body mass of the animals from each experimental group (Table 1 ). Soleus mass and the soleus-to-body mass ratio were reduced 40 and $44 \%$, respectively, after 14 days of nonweight bearing. Intermittent weight bearing had no effect on the absolute mass of the soleus but was effective in attenuating the decline in the soleus-to-body mass ratio. Nevertheless, the soleus-to-body mass ratio for the intermittent weight-bearing group was still $34 \%$ less than the value noted for the weight-bearing animals.

Table 1. Animal and soleus mass

\begin{tabular}{|l|l|l|l|}
\hline Variable & WB Group & NWB Group & IWB Group \\
\hline$n$ & 8 & 7 & 8 \\
\hline Body mass, g & $356 \pm 6$ & $383 \pm 12$ & $361 \pm 11$ \\
\hline Soleus mass, g & $177 \pm 7$ & $107 \pm 7_{-}^{*}$ & $118 \pm 5_{-}^{*}$ \\
\hline Soleus/body mass, $\mathrm{mg} / \mathrm{g}$ & $0.50 \pm 0.02$ & $0.28 \pm 0.01_{-}^{*}$ & $0.33 \pm 0.01_{-}^{*+}$ \\
\hline
\end{tabular}

Values are means $\pm S E ; n$, no. of animals. WB, weight bearing; NWB, non-weight bearing; IWB, intermittent weight bearing.

$\cdot P<0.05$ vs. WB mean. 
$P<0.05$ vs. NWB mean.

\section{Force-velocity-power relationships.}

The majority of single fibers isolated from soleus muscles of the weight-bearing, non-weight-bearing, and intermittent weight-bearing animals expressed type I MHC (27). The present results focus solely on this fiber population.

In comparison to the weight-bearing group, non-weight bearing reduced the diameter of type I fibers by $24 \%$, their peak isometric force by $51 \%$, and their peak isometric force per fiber cross-sectional area (normalized force) by $19 \%$. In contrast, the average $V_{\max }$ of the fibers from the non-weight-bearing animals was $66 \%$ greater than the mean value for the control weight-bearing group.

Intermittent weight bearing was ineffective in restoring either peak force or $V_{\text {max }}$ to weight-bearing levels but did attenuate a portion of the change in these variables that occurred with non-weight bearing (Table2). While the diameter and peak isometric force $(\mathrm{mN})$ of the fibers from the intermittent weight-bearing animals were elevated above non-weight-bearing values by 7 and $20 \%$, respectively, they still remained 18 and $41 \%$ less, respectively, than control weight-bearing values. Furthermore, intermittent weight bearing had no significant effect on the decline in normalized force $\left(\mathrm{kN} / \mathrm{m}^{2}\right)$ that occurred with non-weight bearing. Intermittent weight bearing attenuated the rise in $V_{\text {max }}$ that occurred during non-weight bearing only. The $V_{\max }$ of type I fibers from the intermittent weight-bearing group fell approximately midway between the mean values of the weightbearing and non-weight-bearing groups.

Table 2. Fiber diameter and force-velocity-power results

\begin{tabular}{|l|l|l|l|}
\hline Variable & WB Group & NWB Group & IWB Group \\
\hline$n$ & 52 & 37 & 47 \\
\hline Diameter, $\mu \mathrm{m}$ & $71 \pm 1$ & $54 \pm 2_{-}^{*}$ & $58 \pm 1_{-+}^{*+}$ \\
\hline $\mathrm{P}_{\mathrm{o}}, \mathrm{mN}$ & $0.51 \pm 0.01$ & $0.25 \pm 0.02_{-}^{*}$ & $0.30 \pm 0.01_{-}^{*+}$ \\
\hline $\mathrm{P}_{\mathrm{o}} \mathrm{kN} / \mathrm{m}^{2}$ & $130 \pm 3$ & $105 \pm 3_{-}^{*}$ & $113 \pm 3_{-}^{*}$ \\
\hline$a / \mathrm{P}_{\mathrm{o}}$ & $0.063 \pm 0.003$ & $0.054 \pm 0.003$ & $0.067 \pm 0.005$ \\
\hline$V_{\text {max }} \mathrm{FL} / \mathrm{s}$ & $0.88 \pm 0.04$ & $1.46 \pm 0.10_{-}^{*}$ & $1.08 \pm 0.05_{-+}^{*+}$ \\
\hline $\mathrm{P}$ at peak power, $\mathrm{mN}$ & $0.099 \pm 0.003$ & $0.046 \pm 0.004_{-}^{*}$ & $0.058 \pm 0.002_{-}^{*+}$ \\
\hline $\mathrm{P}$ at peak power, $\mathrm{kN} / \mathrm{m}^{2}$ & $25 \pm 1$ & $19 \pm 1_{-}^{*}$ & $22 \pm 1_{-}^{*+}$ \\
\hline$V$ at peak power, $\mathrm{FL} / \mathrm{s}$ & $0.16 \pm 0.01$ & $0.25 \pm 0.01_{-}^{*}$ & $0.20 \pm 0.01_{+}^{*+}$ \\
\hline Peak power, $\mu \mathrm{N} \cdot \mathrm{FL} \cdot \mathrm{s}^{-1}$ & $16.0 \pm 0.6$ & $10.7 \pm 0.7_{-}^{*}$ & $11.3 \pm 0.4_{-}^{*}$ \\
\hline Peak power, $\mathrm{kN} \cdot \mathrm{m}^{-1} \cdot \mathrm{FL} \cdot \mathrm{s}^{-1}$ & $4.09 \pm 0.17$ & $4.64 \pm 0.20$ & $4.28 \pm 0.15$ \\
\hline
\end{tabular}

Values are means $\pm \mathrm{SE} ; n$, no. of fibers. $\mathrm{P}_{\mathrm{o}}$, peak isometric force; $a / \mathrm{P}_{\mathrm{o}}$, curvature of force of force-velocity relationship, where $a$ is a constant with dimensions of force; $V_{\max }$, velocity axis intercept of force-velocity relationship; $\mathrm{FL}$, fiber lengths; $\mathrm{P}$, force; $V$, shortening velocity;

$\cdot P<0.05$ vs. WB mean.

$+P<0.05$ vs. NWB mean.

Changes in peak force and $V_{\max }$ can be readily observed in the composite force-velocity relationships in Fig.1. At relative forces less than $\sim 50 \%$ of peak force, fibers from the non-weight-bearing animals shortened at velocities substantially greater than did fibers from weight-bearing animals. The force-velocity relationship for the intermittent weight-bearing group appeared to fall midway between the relationships of the other two groups (Fig. 1 $A$ ). Differences in peak force between the three groups can be readily observed when force-velocity relationships were plotted on an absolute force axis (Fig. $1 B$ ). Figure 1illustrates that at absolute forces between 
$\sim 0.05 \mathrm{mN}$ and peak isometric force, fibers from both the non-weight-bearing and intermittent weight-bearing groups shortened considerably slower than did fibers from the weight-bearing group. However, the forcevelocity relationships for the fibers obtained from the intermittent weight-bearing and non-weight-bearing groups crossed over the weight-bearing curve at low absolute loads. Therefore, at these low loads, fibers from the intermittent weight-bearing and non-weight-bearing animals shortened at velocities that exceeded the shortening velocity of the fibers from the weight-bearing animals. This rise in velocity was greatest for the nonweight-bearing group.
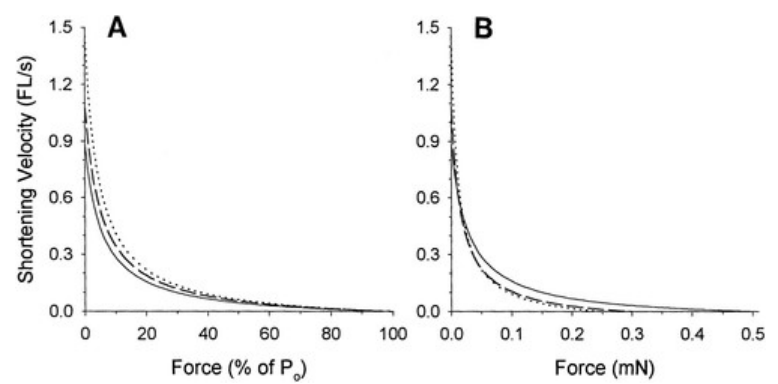

Fig. 1. Force-velocity relationships of type I fibers from each experimental group. $A$ : relative force scale. $B$ : absolute force scale. $P_{o}$, peak isometric force; FL, fiber lengths. Solid line, weight-bearing group; dotted line, non-weightbearing group; dashed line, intermittent weight-bearing group. No. of fibers per group is same as in Table $\underline{2}$.

Neither non-weight bearing nor intermittent weight bearing affecteda/ $\mathrm{P}_{0}$, which specifies the curvature of the force-velocity relationship (Table2). Consequently, there were no differences in the relative load associated with the peak power output of type I fibers from each experimental group $(19 \pm 0.3,18 \pm 0.4$, and $19 \pm 0.5 \%$ of peak isometric force for the weight-bearing, non-weight-bearing, and intermittent weight-bearing groups, respectively; $P>0.05)$. However, because non-weight bearing and intermittent weight bearing both reduced peak isometric force while elevating $V_{\max }$ in comparison to the weight-bearing condition, the absolute forces and shortening velocities associated with peak power output differed between the three treatments. Fibers from the non-weight-bearing group produced $54 \%$ less absolute force and $24 \%$ less normalized force at peak power output than did fibers from the weight-bearing animals. Compared with the non-weight-bearing condition, the intermittent weight-bearing treatments served to elevate both the absolute (26\%) and normalized force (16\%) produced at peak power. However, this countermeasure did not restore these forces to weight-bearing levels because fibers from the intermittent weight-bearing group still produced $41 \%$ less absolute force and $12 \%$ less force per fiber cross-sectional area at peak power output than fibers from the weight-bearing animals.

At the submaximal force associated with peak power output, non-weight-bearing type I fibers shortened on average $56 \%$ faster than type I fibers from the weight-bearing group. Intermittent weight bearing served to reduce this non-weight-bearing-induced rise in shortening velocity by $\sim 45 \%$. However, type I fibers obtained from the intermittent weight-bearing rats still shortened at a velocity that exceeded the shortening velocity of type I fibers from the weight-bearing animals by $25 \%$.

Non-weight bearing reduced the average peak absolute power output $\left(\mu \mathrm{N} \cdot \mathrm{FL} \cdot \mathrm{s}^{-1}\right)$ of type I soleus fibers by 33\% (Table 2). Intermittent weight bearing had no significant effect on this non-weight-bearing-induced reduction in peak absolute power. Figure 2illustrates that at essentially all relative forces (Fig. $2 A$ ), type I fibers from both the non-weight-bearing and intermittent weight-bearing groups produced substantially less power than did type I fibers from the weight-bearing animals. These differences in power output were even more evident when the force-power results were plotted on an absolute force axis (Fig.2B). 

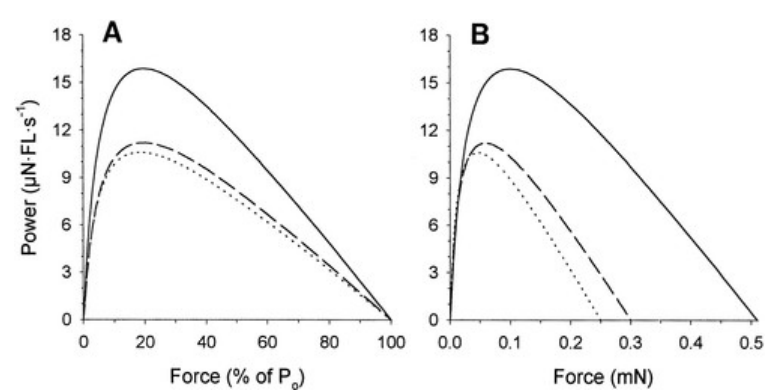

Fig. 2. Force-power relationships of type I fibers from each experimental group. $A$ : relative force scale. $B$ : absolute force scale. Solid line, weight-bearing group; dotted line, non-weight-bearing group; dashed line, intermittent weightbearing group. No. of fibers per group is same as in Table $\underline{2}$.

When peak power was normalized for differences in fiber size $\left(\mathrm{kN} \cdot \mathrm{m}^{-2} \cdot \mathrm{FL} \cdot \mathrm{s}^{-1}\right)$, single fibers from the nonweight-bearing animals produced slightly greater peak power than did fibers from the intermittent weightbearing group, which in turn produced slightly greater peak power than did fibers from the weight-bearing rats. However, these differences were not statistically significant.

\section{Force-pCa relationships.}

Force-pCa relationships (Fig. $\underline{3}$ ) and Hill plot analysis (Table $\underline{3}$ ) were used to assess the dependence of force production on free $\mathrm{Ca}^{2+}$ levels in single fibers. Non-weight bearing shifted the force-pCa relationship to the right so that a significantly greater free $\mathrm{Ca}^{2+}$ concentration was required to achieve one-half maximal activation. In all groups, there was a steeper rise in force at free $\mathrm{Ca}^{2+}$ concentrations eliciting $<50 \%$ of peak force $\left(n_{2}\right)$ in comparison to levels of $\mathrm{Ca}^{2+}$ activation producing $>50 \%$ of relative force $\left(n_{1}\right)$. Non-weight bearing had no effect on the slope of the Hill plots for data below or above $50 \%$ of peak force, nor did this treatment affect the free $\mathrm{Ca}^{2+}$ concentration required for the onset of force (activation threshold). Compared with fibers from the nonweight-bearing animals, intermittent weight bearing shifted the force-pCa relationship to the left. Nevertheless, at submaximal forces $<50 \%$ of peak force, the force-pCa relationship for fibers from the intermittent weightbearing animals fell between the curves observed for fibers from the weight-bearing and non-weight-bearing groups. Hill plot analysis indicated that the free $\mathrm{Ca}^{2+}$ concentration required for one-half maximal activation was statistically similar for fibers obtained from the weight bearing and intermittent weight-bearing animals.

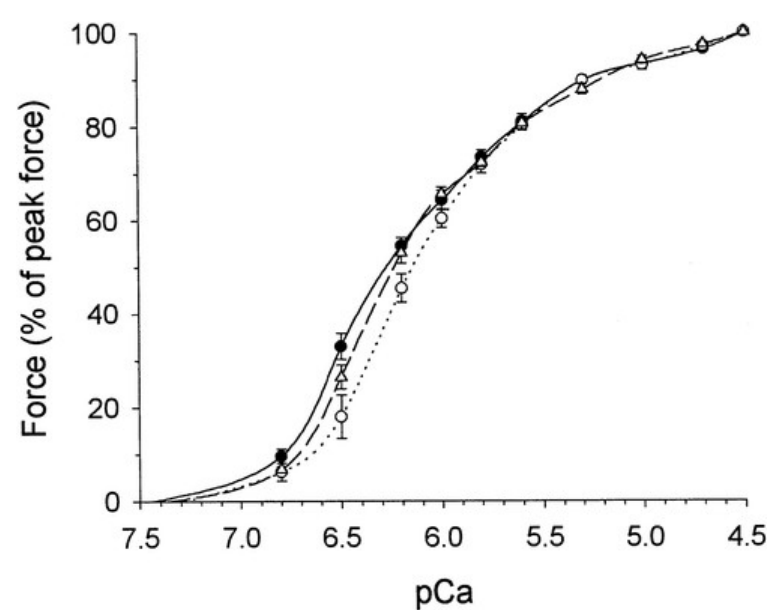

Fig. 3. Force-pCa relationships of type I soleus fibers from each experimental group. Symbols represent mean values with error bars denoting $\pm 1 \mathrm{SE}$. Solid line, weight-bearing group; dotted line, non-weight-bearing group; dashed line, intermittent weight-bearing group. No. of fibers per group is same as in Table $\underline{3}$.

Table 3. Force-pCa results

\begin{tabular}{|l|l|l|l|}
\hline Variable & WB Group & NWB Group & IWB Group \\
\hline
\end{tabular}




\begin{tabular}{|l|l|l|l|}
\hline$n$ & 11 & 11 & 16 \\
\hline Activation threshold, $\mu \mathrm{M} \mathrm{Ca}^{2+}$ & $0.036 \pm 0.005$ & $0.053 \pm 0.008$ & $0.045 \pm 0.004$ \\
\hline Half-maximal activation, $\mu \mathrm{M} \mathrm{Ca}^{2+}$ & $0.497 \pm 0.040$ & $0.676 \pm 0.063 \underline{-150}$ & $0.537 \pm 0.035 \underline{3-151}$ \\
\hline$n_{1}$ & $0.97 \pm 0.06$ & $1.08 \pm 0.09$ & $0.96 \pm 0.05$ \\
\hline$n_{2}$ & $2.28 \pm 0.13$ & $2.28 \pm 0.14$ & $2.38 \pm 0.12$ \\
\hline
\end{tabular}

Values are means $\pm \mathrm{SE} ; n$, no. of fibers. $n_{1}$ and $n_{2}$, slopes of Hill plots for values $>$ and $<$ half-maximal activation, respectively.

${ }^{\mathrm{F} 3}-150 P<0.05$ vs. WB mean.

${ }^{F 3-151} P<0.05$ vs. NWB mean.

\section{DISCUSSION}

\section{Isotonic contractile properties.}

In agreement with McDonald et al. (16), 14 days of non-weight bearing induced significant alterations in the isotonic contractile properties of type I fibers obtained from the rat soleus. Most important from a functional point of view was the significant reduction in the absolute peak power output of these fibers. Type I fibers from the non-weight-bearing animals produced $>30 \%$ less peak power than did fibers from the weight-bearing group despite the fact that under conditions associated with peak power output, the former fibers shortened at a $56 \%$ greater velocity. An increase in shortening velocity is beneficial in terms of power output, particularly in this instance, where it can partially compensate for the reduced ability of the type I fibers from the non-weightbearing rats to produce force $(\underline{2}, \underline{16})$. However, the fibers obtained from the non-weight-bearing group, primarily as a result of their smaller size, produced $54 \%$ less absolute force than did fibers from the weight-bearing animals at peak power output. Thus, at peak power, the fibers from the non-weight-bearing group shortened at 1.54 times the velocity $(V)$ but produced only 0.46 times the absolute force $(P)$ of fibers from the weight-bearing animals. Peak power, the product of $\mathrm{P}$ and $\mathrm{V}$, or $0.46 \mathrm{P} \cdot 1.54 \mathrm{~V}$, was $\sim 70 \%$ of the weight-bearing mean.

A major finding of this study was that intermittent weight-bearing treatments, totaling $40 \mathrm{~min} /$ day of standing weight-bearing activity, had no significant effect on this decline in peak absolute power output. Although intermittent weight bearing partially attenuated the fiber atrophy that occurs during non-weight bearing and by doing so increased the submaximal force associated with peak power output ( $26 \%$ vs. non-weight-bearing mean), this treatment also reduced the shortening velocity of these fibers at peak power (-20\% vs. non-weightbearing mean). Because these changes in fiber force production and shortening velocity have opposing effects on peak power, the average peak power output of type I fibers after the intermittent weight-bearing treatments remained at the non-weight-bearing level.

These results have important implications concerning the effectiveness of intermittent weight bearing as a countermeasure to non-weight-bearing-induced soleus atrophy. Preliminary results from our laboratory indicate that 17 days of non-weight bearing reduce the peak power of human type I soleus fibers (25). While it is not entirely clear as to whether a loss of soleus power would hamper the physical performance of humans during the microgravity conditions of spaceflight, these reductions would be expected to limit work capacity on a return to a gravity environment (4). In the case of bed rest, it is likely that the ability to resume a normal lifestyle on the resumption of ambulatory activity would be compromised by a loss of muscle power. The inability of intermittent weight-bearing treatments to reduce non-weight-bearing-induced losses in peak power brings into question the practical effectiveness of this countermeasure. What appears to be required is a modified procedure that1) restores peak force to a greater extent than the present protocol and/or2) does not affect the elevation in type I fiber shortening velocity that occurs during non-weight bearing. 


\section{Fiber Ca2+sensitivity.}

Fiber $\mathrm{Ca}^{2+}$ sensitivity is thought to be regulated by differences in thin filament regulatory protein isoforms ( $\underline{12}$, 21) and the kinetics of cross-bridge attachment and detachment (1). In addition to these regulatory mechanisms, the relationship between force and $\mathrm{Ca}^{2+}$ concentration is highly sensitive to the spacing between the thin and thick myofilaments (11). In agreement with previous results $(\underline{8}, \underline{18}), 14$ days of non-weight bearing reduced the $\mathrm{Ca}^{2+}$ sensitivity of single fibers. This is evident by the rightward shift of the force-pCa relationship for forces $<50 \%$ of peak force and by the significantly greater free $\mathrm{Ca}^{2+}$ concentration required to attain half-maximal activation. The mechanism(s) responsible for this rightward shift in the force-pCa relationship is not presently known. Non-weight bearing is associated with an increase in the fast isoforms of troponin T (TnT) and troponin I $(\underline{3})$. Single intermediate- and fast-velocity fibers expressing a fast $\mathrm{TnT}$ isoform have a lower $\mathrm{Ca}^{2+}$ sensitivity than do slow-velocity fibers expressing the slow TnT isoform (12). However, it is not known what effect fast TnT expression in a slow fiber would have on the force-pCa relationship. Electron micrographs demonstrating a disproportionate loss of thick filaments $(\underline{24})$ raise the possibility that the ultrastructural geometry of the myofilament lattice could be altered during non-weight bearing. While it is not known whether changes in filament geometry actually occur, an increase in the distance between thin and thick filaments, and/or a change in the angle at which myosin binds to actin, is consistent with a rightward shift in the force-pCa relationship (111).

Based on the Hill plot analysis results in Table 3 , one could conclude that intermittent weight-bearing treatments restored fiber $\mathrm{Ca}^{2+}$ sensitivity to the weight-bearing level. However, an examination of the force-pCa relationships (Fig. $\underline{3}$ ) indicates that at forces $<50 \%$ of peak force, the relationship for fibers from the intermittent weight-bearing animals fell between the relationships observed for the other two groups. Taken together, these results indicate that fiber $\mathrm{Ca}^{2+}$ sensitivity may not have been completely restored by the intermittent weightbearing treatments. If changes in the expression of thin filament regulatory proteins are responsible for the rightward shift in the force-pCa relationship after non-weight bearing, then the intermittent weight-bearing treatments may have worked to partially reduce the expression of these isoforms. Alternatively, if the rightward shift was due to changes in lattice spacing, intermittent weight bearing may have attenuated these changes in ultrastructural geometry. This second hypothesis is attractive because it could also explain the reduction in fiber $V_{\max }$ and the increase in peak normalized force that occurred with the intermittent weight-bearing treatments (20).

\section{Conclusions.}

The stimulus provided by intermittent weight-bearing treatments, which is presumed to be the restoration of normal weight-bearing activity by the hindlimb extensor muscles, attenuated a non-weight-bearing-induced reduction in fiber $\mathrm{Ca}^{2+}$ sensitivity. However, this countermeasure had no effect on the decline in type I fiber peak power output that occurs during 14 days of non-weight bearing. Intermittent weight bearing was ineffective in this regard because it had opposing effects on fiber force (an increase vs. the non-weight-bearing mean) and fiber shortening velocity (a decrease vs. the non-weight-bearing mean). This inability to attenuate reductions in type I peak power output is a shortcoming of intermittent weight bearing as a countermeasure to non-weightbearing-induced soleus atrophy.

This study was supported by a Wisconsin Space Grant Consortium scholarship to J. J. Bangart, National Aeronautics and Space Administration (NASA) Postdoctoral Space Biology Research Associate Award SBRA93-06 to J. J. Widrick, and NASA Grants NAG2-212 and NAGW-4376 to R. H. Fitts.

\section{AUTHOR NOTES}

Address for reprint requests: R. H. Fitts, Marquette Univ., Dept. of Biology, Wehr Life Sciences Bldg., P.O. Box 1881, Milwaukee, WI 53201-1881. 


\section{REFERENCES}

1. Brandt P. W., Cox R. N., Kawai M., Robinson T.Regulation of tension in skinned muscle fibers. Effect of crossbridge kinetics on apparent Ca2+ sensitivity.J. Gen. Physiol. 7919829971016

2. Caiozzo V. J., Baker M. J., Herrick R. E., Tao M., Baldwin K. M.Effect of spaceflight on skeletal muscle: mechanical properties and myosin isoform content of a slow muscle.J. Appl. Physiol.76199417641773

3. Campione M., Ausoni S., Guezennec C. Y., Schiaffino S.Myosin and troponin changes in rat soleus muscle after hindlimb suspension.J. Appl. Physiol.74199311561160

4. Convertino V. A.Physiological adaptations to weightlessness: effects on exercise and work performance.Exercise and Sport Sciences Reviews, Pandolf K. B., Holloszy J. O.181990119166Williams \& WilkinsBaltimore, MD

5. D'Aunno D. S., Robinson R. R., Smith G. S., Thomason D. B., Booth F. W.Intermittent acceleration as a countermeasure to soleus muscle atrophy.J. Appl. Physiol.721992428433

6. Dudley G. A., Duvoisin M. R., Convertino V. A., Buchanan P.Alterations of the in vivo torque-velocity relationship of human skeletal muscle following 30 days exposure to simulated microgravity.Aviat. Space Environ. Med.601989659663

7. Fabiato A., Fabiato F.Calculator programs for computing the composition of the solutions containing multiple metals and ligands used for experiments in skinned muscle cells.J. Physiol. (Paris)751979463505

8. Gardetto P. R., Schluter J. M., Fitts R. H.Contractile function of single muscle fibers after hindlimb suspension.J. Appl. Physiol.66198927392749

9. Giulian G. G., Moss R. L., Greaser M.Improved methodology for analysis and quantitation of proteins on onedimensional silver-stained slab gels.Anal. Biochem.1291983277287

10. Godt R. E., Lindley B. D.Influence of temperature upon contractile activation and isometric force production in mechanically skinned muscle fibers of the frog.J. Gen. Physiol. 801982279297

11. Godt R. E., Maughan D. W.Influence of osmotic compression on calcium activation and tension in skinned muscle fibers of the rabbit.Pflügers Arch.3911981334337

12. Greaser M. L., Moss R. L., Reiser P. J.Variations in contractile properties of rabbit single muscle fibres in relation to troponin T isoforms and myosin light chains.J. Physiol. (Lond.) 40619888598

13. Grigoryeva L. S., Kozlovskaya I. B.Effect of weightlessness and hypokinesia on velocity and strength properties of human muscles.Kosm. Biol. Aviakosm. Med.2119872730

14. Hill A. V.The heat of shortening and the dynamic constants of muscle.Proc. R. Soc. Lond. B Biol. Sci.1261938136195

15. Julian F. J., Moss R. L.Effects of calcium and ionic strength on shortening velocity and tension development in frog skinned muscle fibres.J. Physiol. (Lond.)3111981179199

16. McDonald K. S., Blaser C. A., Fitts R. H.Force-velocity and power characteristics of rat soleus muscle fibers after hindlimb suspension.J. Appl. Physiol.77199416091616

17. McDonald K. S., Fitts R. H.Effect of hindlimb unweighting on single soleus fiber maximal shortening velocity and ATPase activity.J. Appl. Physiol.74199329492957

18. McDonald K. S., Fitts R. H.Effect of hindlimb unloading on rat soleus fiber force, stiffness, and calcium sensitivity.J. Appl. Physiol.79199517961802

19. Metzger J. M., Moss R. L.Greater hydrogen ion-induced depression of tension and velocity in skinned single fibres of rat fast than slow muscles.J. Physiol. (Lond.)3931987727742

20. Metzger J. M., Moss R. L.Shortening velocity in skinned single muscle fibers. Influence of filament lattice spacing.Biophys. J.521987127131

21. Moss R. L., Lauer M. R., Giulian G. G., Greaser M. L.Altered Ca² dependence of tension development in skinned skeletal muscle fibers following modification of troponin by partial substitution with cardiac troponin C.J. Biol. Chem.261198660966099

22. Pierotti D. J., Roy R. R., Flores V., Edgerton V. R.Influence of 7 days of hindlimb suspension and intermittent weight support on rat muscle mechanical properties.Aviat. Space Environ. Med.611990205210 
23. Reiser P. J., Kasper C. E., Moss R. L.Myosin subunits and contractile properties of single fibers from hypokinetic rat muscles.J. Appl. Physiol.63198722932300

24. Riley D. A., Slocum G. R., Bain J. L. W., Sedlak F. R., Sowa T. E., Mellender J. W.Rat hindlimb unloading: soleus histochemistry, ultrastructure, and electromyography.J. Appl. Physiol.6919905866

25. Romatowski J. G., Widrick J. J., Sherwood J., Bangart J. J., Costill D. L., Fitts R. H.Isotonic contractile properties of soleus muscle fibers after 17 days of bedrest (Abstract).Med. Sci. Sports Exercise281996S146

26. Thomason D. B., Herrick R. E., Baldwin K. M.Activity influences on soleus muscle myosin during rodent hindlimb suspension.J. Appl. Physiol.631987138144

27. Widrick J. J., Bangart J. J., Karhanek M., Fitts R. H.Soleus fiber force and maximal shortening velocity after non-weight bearing with intermittent activity.J. Appl. Physiol.801996981987

28. Widrick J. J., Romatowski J. G., Karhanek M., Fitts R. H.Contractile properties of rat, rhesus monkey, and human type I muscle fibers.Am. J. Physiol.272Regulatory Integrative Comp. Physiol. 411997R34R42 